\title{
Subcutaneous thigh fat area is unrelated to risk of type 2 diabetes in a prospective study of Japanese Americans
}

\author{
D. Hoyer • E. J. Boyko • M. J. McNeely • \\ D. L. Leonetti • S. E. Kahn • W. Y. Fujimoto
}

Received: 9 May 2011 / Accepted: 18 July 2011 /Published online: 12 August 2011

(C) Springer-Verlag (outside the USA) 2011

\begin{abstract}
Aims/hypothesis Cross-sectional research has reported a negative association between subcutaneous thigh fat (STF) and type 2 diabetes prevalence but no prospective research on this association exists using direct measurements of STF obtained from imaging studies while adjusting for other fat depots. We studied the independent associations of intraabdominal fat (IAF), subcutaneous abdominal fat (SAF) and STF with future risk of diabetes.

Methods We prospectively followed 489 non-diabetic Japanese Americans (BMI 25.0-29.9 kg/m² 32.7\%, $\geq 30.0 \mathrm{~kg} / \mathrm{m}^{2} 5.4 \%$ ) over 10 years for the development of diabetes defined by use of hypoglycaemic medication or a fasting plasma glucose $\geq 7.0 \mathrm{mmol} / 1$ or $2 \mathrm{~h} \geq 11.1 \mathrm{mmol} /$ 1 during an OGTT. STF, SAF and IAF area were measured by computed tomography scan and mid-thigh circumference (TC) by tape measure at baseline.

Results Over 10 years, 103 people developed diabetes. STF area was not independently associated with the odds of
\end{abstract}

D. Hoyer

School of Public Health, University of Washington,

Seattle, WA, USA

E. J. Boyko $(\bowtie) \cdot$ S. E. Kahn

VA Puget Sound Health Care System,

1100 Olive Way, Suite 1400,

Seattle, WA 98101, USA

e-mail: eboyko@uw.edu

E. J. Boyko • M. J. McNeely • S. E. Kahn • W. Y. Fujimoto Department of Medicine, University of Washington,

Seattle, WA, USA

D. L. Leonetti

Department of Anthropology, University of Washington,

Seattle, WA, USA developing diabetes in a univariate or multiple logistic regression model (OR for a $1 \mathrm{SD}$ increase 0.8 [95\% CI 0.5 , 1.2]) adjusted for age, sex, BMI, IAF and SAF. The only fat depot associated with diabetes odds in this model was IAF. TC was borderline significantly associated with a lower odds of developing diabetes $(0.7$ [95\% CI 0.5, 1.0], $p=0.052)$.

Conclusions/interpretation Similar to other research, TC was negatively associated with diabetes risk, whereas STF was not, arguing that the negative association between TC and diabetes observed in other research is not due to STF mass. IAF area emerged as the only measured fat depot that was independently associated with type 2 diabetes risk.

Keywords Adiposity · Computed tomography · Diabetes mellitus · Epidemiology · Japanese American · Prospective study - Thigh circumference . Thigh subcutaneous fat . Visceral fat . Waist circumference
Abbreviations
CT Computed tomography
IAF Intra-abdominal fat
SAF Subcutaneous abdominal fat
STF Subcutaneous thigh fat
TC Thigh circumference

Cross-sectional research has shown a negative association between subcutaneous thigh fat (STF) and thigh circumference (TC) and glucose concentrations, dyslipidaemia and type 2 diabetes [1-3]. Investigators have shown that greater leg fat mass may be associated with lower prevalence of diabetes mellitus in obese participants $[4,5]$. A potential causal role for thigh fat in the development of type 2 diabetes has also been suggested by a prospective population-based study that demonstrated that the incidence 
of this outcome was negatively associated with TC and positively associated with waist circumference [6]. It is well recognised that pathological conditions that are accompanied by loss of gluteo-femoral fat such as glucocorticoid excess or partial lipodystrophy are associated with metabolic abnormalities [7]. Although these findings support a potential protective effect of thigh fat in the development of type 2 diabetes, other results do not support this association. A prospective investigation in Pima Indians showed that TC was less informative than waist circumference or generalised adiposity measures in predicting the risk of type 2 diabetes [8], and no significant association was seen between STF depot size measured by computed tomography (CT) and type 2 diabetes in one cross-sectional study [9]. No prospective investigation exists to our knowledge on the association between directly measured thigh fat and risk of type 2 diabetes in normal, overweight or obese individuals.

Other fat depots have also been associated with type 2 diabetes risk. A positive association between intra-abdominal fat (IAF) and type 2 diabetes prevalence and incidence has been shown in cross-sectional and prospective studies [9-12]. A less consistent association has been observed between subcutaneous abdominal fat (SAF) and the presence of metabolic risk factors or type 2 diabetes [9-12]. These data argue for differential associations between body fat and diabetes depending on fat location.

In this study we aimed to determine longitudinally the relationship between STF, measured by $\mathrm{CT}$, and the development of type 2 diabetes while adjusting for potential confounding by other significant fat depots.

\section{Methods}

Study participants The study population comprised 519 non-diabetic Japanese-American men and women enrolled in the Japanese-American Community Diabetes Study. All individuals were of $100 \%$ Japanese ancestry. The details on the selection criteria and recruitment of individuals for this study have been previously published [13]. Briefly, participants were chosen as volunteers from a community-wide mailing list and telephone directory that included nearly $95 \%$ of the Japanese-American population in King County, WA, USA. This population was followed prospectively over 10 years. Individual diabetes status was assessed at baseline and at 2.5, 5 and 10 year follow-up visits. Thirty participants did not attend any of the follow-up visits due to death, inability to locate them or withdrawal from the study, leaving a total of 489 participants who were included in this analysis. This study received approval from the University of Washington Human Subjects Office and all participants provided written informed consent to participate.
Measurements Evaluations were done at the General Clinical Research Center at the University of Washington. All research protocols used were reviewed and approved by the institution's Human Subjects Review Committee and informed consent was obtained from all participants. BMI was computed as weight in kilograms divided by height in metres squared $\left(\mathrm{kg} / \mathrm{m}^{2}\right)$. Waist circumference was measured at the umbilicus in centimetres using a tape measure.

Normal glucose tolerance (NGT), impaired glucose tolerance (IGT) or type 2 diabetes classification was determined using a $75 \mathrm{~g}$ OGTT following a $10 \mathrm{~h}$ fast. Diabetes status was defined as use of oral hypoglycaemic medication or insulin, or fasting plasma glucose $\geq 7.0 \mathrm{mmol} / \mathrm{l}$ or $2 \mathrm{~h}$ glucose $\geq 11.1 \mathrm{mmol} / \mathrm{l}$. Participants were considered to have developed diabetes during follow-up if this outcome occurred at the $2.5,5$ or 10 year follow-up time points. Plasma glucose was assayed by an automated glucose oxidase method. Family history of diabetes was considered positive if any first-degree relative had diabetes [13].

Single $(1 \mathrm{~cm})$ CT scan slices were obtained at the abdomen (umbilicus level) and thigh (halfway between the greater trochanter and the superior margin of the patella) [14]. Cross-sectional measurements of fat area (square centimetres) were obtained from these on each participant as follows: SAF, IAF (within the confines of the transversalis fascia) and left STF [14]. The intra-observer variability for multiple measurements by a single observer of a single CT scan ranged from $0.2 \%$ to $1.4 \%$. The point at which the mid-thigh CT was obtained was marked and the $\mathrm{TC}$ was assessed at this point using a tape measure.

Statistical analysis Multiple logistic regression models were created to estimate the relationship between fat depots and diabetes occurrence while adjusting for other variables considered potential confounders. The variance inflation factor was used to identify a high likelihood of collinearity in multivariable models if it exceeded 4. ORs for all continuous variables were calculated for a $1 \mathrm{SD}$ increase, with SD defined as the square root of the variance. The presence of interactions between sex and fat depots and sex and BMI was tested by insertion of first order interaction terms into the regression models. IBM Statistical Package for the Social Sciences (SPSS) Statistics 18 (Armonk, NY, USA) was used for statistical analyses.

\section{Results}

Over the follow-up period, $103(19.8 \%)$ of the 489 participants developed diabetes. At baseline, study participants had a mean BMI of $24.1 \mathrm{~kg} / \mathrm{m}^{2}$, a mean age of 52.2 years, $51.5 \%$ were men and $37.6 \%$ of individuals had a family history of diabetes (Table 1). Although the average 
Table 1 Characteristics of study participants at baseline by diabetes development during follow-up

\begin{tabular}{|c|c|c|c|c|}
\hline Characteristic & Study population & Did not progress to diabetes $(n=386)$ & Progressed to diabetes $(n=103)$ & $p$ value \\
\hline Age (years) & $52.2(12.0)$ & $51.2(11.8)$ & $57.9(10.9)$ & $<0.001$ \\
\hline Male, $n(\%)$ & $265(51.5)$ & $197(51.0)$ & $56(54.4)$ & 0.533 \\
\hline Fasting glucose $(\mathrm{mmol} / \mathrm{l})$ & $5.2(0.6)$ & $5.1(0.5)$ & $5.6(0.5)$ & $<0.001$ \\
\hline Positive family history of diabetes, $n(\%)$ & $195(37.6)$ & $123(31.9)$ & $57(55.3)$ & $<0.001$ \\
\hline BMI $\left(\mathrm{kg} / \mathrm{m}^{2}\right)$ & $24.1(3.2)$ & $23.8(3.1)$ & $25.3(3.6)$ & $<0.001$ \\
\hline Waist circumference $(\mathrm{cm})$ & $86.0(8.7)$ & $85.1(8.7)$ & $89.3(8.0)$ & $<0.001$ \\
\hline $\operatorname{STF}\left(\mathrm{cm}^{2}\right)$ & $65.0(32.0)$ & $65.2(32.0)$ & $63.0(30.5)$ & 0.522 \\
\hline $\operatorname{IAF}\left(\mathrm{cm}^{2}\right)$ & $81.4(49.6)$ & $75.1(46.1)$ & $109.5(51.9)$ & $<0.001$ \\
\hline $\operatorname{SAF}\left(\mathrm{cm}^{2}\right)$ & $157.3(76.4)$ & $153.2(78.1)$ & $173.2(70.2)$ & 0.022 \\
\hline Mid-TC (cm) & $49.3(4.6)$ & $49.3(4.2)$ & $49.0(5.7)$ & 0.382 \\
\hline
\end{tabular}

Data are means (SD) or $n(\%)$

BMI in this population was in the category usually defined as normal (BMI $<25.0 \mathrm{~kg} / \mathrm{m}^{2}$ ), BMI ranged from 16.6 to $36.9 \mathrm{~kg} / \mathrm{m}^{2}$, with $61.9 \%$ of persons having a BMI $<25.0$ $\mathrm{kg} / \mathrm{m}^{2}, 32.7 \%$ between 25.0 and $29.9 \mathrm{~kg} / \mathrm{m}^{2}$ and $5.4 \%$ at $30.0 \mathrm{~kg} / \mathrm{m}^{2}$ or greater. Ranges of thigh measurements were as follows: mid-TC $36.2-63 \mathrm{~cm}$; subcutaneous fat area 1.8$215.5 \mathrm{~cm}^{2}$. Baseline characteristics by the development of diabetes during follow-up are presented in Table 1. All measures of overall and regional adiposity and circumference measures were positively and significantly correlated with each other except for a non-significant correlation between IAF and STF (Table 2).

Logistic regression analysis was performed to estimate the bivariate association between development of diabetes over the 10 year follow-up and each of the independent variables. Age, BMI, waist circumference, IAF area, SAF area, fasting glucose and family history of diabetes each showed a significant positive association with the development of diabetes in these analyses, whereas STF area and TC showed an insignificant negative association. Sex was not significantly associated with the development of diabetes (Table 3). Associations between thigh measurements and IAF and diabetes risk were estimated separately by sex. This analysis found a significant positive association between greater IAF area (1 SD increment) and diabetes risk (OR $[95 \% \mathrm{CI}]$ men 2.1 [1.5, 2.8], women $2.0[1.4,2.7])$. The associations between STF area and TC and diabetes risk were non-significant for both men and women (STF OR [95\% CI] men $1.2[0.9,1.6]$, women 0.8 $[0.6,1.2]$; TC men $1.0[0.8,1.4]$, women $0.8[0.5,1.1])$.

Multiple logistic regression models were used to estimate the relationship between thigh fat and the development of type 2 diabetes while adjusting for other independent variables. All models were adjusted for age, sex and family history of diabetes. STF area was not independently associated with the odds of diabetes over 10 years in a model adjusted for age, sex and family history of diabetes (Table 4, model 1). After adjustment for BMI, a negative but statistically non-significant association between STF area and diabetes odds was observed (Table 4, model 2). However, the magnitude of the association between STF area and diabetes odds diminished with the inclusion of IAF and SAF areas in the model (Table 4, model 3). No association was observed between STF and type 2 diabetes odds in any of these models. There was no evidence of collinearity in the multivariable models shown in Table 4 as judged by the variance inflation factor.

IAF area was independently associated with the development of diabetes, whereas SAF area was not in a model that contained both of these measurements (Table 4, model 3). Mid-TC was negatively associated with diabetes incidence (Table 4, model 4) with higher circumference associated with lower odds of diabetes development, but this association was of borderline statistical significance $(p=0.052)$. Insertion of
Table 2 Correlation matrix of measures of overall and regional adiposity displaying Pearson correlation coefficients

All associations were significant at $p<0.001$ except $^{\dagger} p=0.118$

\begin{tabular}{llccccc}
\hline Characteristic & BMI & Waist circumference & Mid-TC & STF area & IAF area & SAF area \\
\hline BMI & 1 & & & & & \\
Waist circumference & 0.84 & 1 & 1 & & & \\
Mid-TC & 0.67 & 0.45 & 0.39 & 1 & & \\
STF area & 0.17 & 0.15 & 0.16 & $-0.07^{\dagger}$ & 1 & \\
IAF area & 0.63 & 0.72 & 0.36 & 0.53 & 0.42 & 1 \\
SAF area & 0.63 & 0.74 & & & & \\
\hline
\end{tabular}


Table 3 Bivariate associations with incidence of diabetes over 10 years among Japanese Americans

\begin{tabular}{llr}
\hline Characteristic & OR $(95 \% \mathrm{CI})$ & $p$ value \\
\hline Age (years) & $1.9(1.5,2.4)$ & $<0.001$ \\
Male $(\%)$ & $1.1(0.7,1.8)$ & 0.548 \\
BMI $\left(\mathrm{kg} / \mathrm{m}^{2}\right)$ & $1.5(1.2,1.9)$ & $<0.001$ \\
Waist circumference $(\mathrm{cm})$ & $1.6(1.3,2.0)$ & $<0.001$ \\
STF $\left(\mathrm{cm}^{2}\right)$ & $0.9(0.7,1.2)$ & 0.532 \\
IAF $\left(\mathrm{cm}^{2}\right)$ & $2.0(1.6,2.5)$ & $<0.001$ \\
SAF $\left(\mathrm{cm}^{2}\right)$ & $1.3(1.0,1.6)$ & 0.022 \\
Fasting glucose (mmol/l) & $2.9(2.2,3.8)$ & $<0.001$ \\
Family history of diabetes $(\%)$ & $2.7(1.7,4.1)$ & $<0.001$ \\
Mid-TC (cm) & $0.9(0.7,1.1)$ & 0.331 \\
\hline
\end{tabular}

ORs for continuous variables reflect a $1 \mathrm{SD}$ magnitude increase

IAF as an additional covariate into Table 4, model 4 resulted in a diminution in the association between mid-TC and diabetes development (OR [95\% CI] 0.75 [0.5, 1.1], $p=$ 0.183 ). Insertion of waist circumference into the regression models shown in Table 4 in place of BMI did not result in a change in the statistically significant association between diabetes development and IAF area, or the non-significant

Table 4 Adjusted models of the incidence of diabetes over 10 years in relation to body composition among Japanese Americans

\begin{tabular}{lllr}
\hline Model & Variable & OR $(95 \%$ CI $)$ & $p$ value \\
\hline 1 & Age & $1.9(1.5,2.7)$ & $<0.001$ \\
& Family history & $2.5(1.6,4.0)$ & $<0.001$ \\
& Male & $1.4(0.8,2.6)$ & 0.241 \\
STF & $1.1(0.8,1.5)$ & 0.551 \\
& Age & $1.7(1.2,2.3)$ & $<0.001$ \\
& Family history & $2.3(1.4,3.7)$ & $<0.001$ \\
Male & $0.6(0.3,1.2)$ & 0.150 \\
& BMI & $1.8(1.4,2.4)$ & $<0.001$ \\
& STF & $0.7(0.5,1.1)$ & 0.108 \\
& Age & $1.6(1.2,2.2)$ & 0.001 \\
& Family history & $2.3(1.4,3.6)$ & 0.001 \\
& Male & $0.5(0.2,1.1)$ & 0.087 \\
BMI & $1.6(1.0,2.6)$ & 0.038 \\
& STF & $0.8(0.5,1.2)$ & 0.279 \\
& IAF & $1.5(1.1,2.1)$ & 0.033 \\
SAF & $0.8(0.5,1.2)$ & 0.286 \\
& Age & $2.1(1.2,3.7)$ & 0.007 \\
& Family history & $2.2(1.3,3.4)$ & 0.001 \\
Male & $1.1(0.7,1.7)$ & 0.831 \\
& BMI & $2.1(1.5,3.0)$ & $<0.001$ \\
Mid-TC & $0.7(0.5,1.0)$ & 0.052 \\
\hline & & &
\end{tabular}

ORs for continuous variables reflect a $1 \mathrm{SD}$ magnitude increase associations between this outcome and both STF area and mid-TC (data not shown).

To assess whether the results of the models in Table 4 differed by sex, first order interactions between sex and fat depots and sex and BMI were tested in all models in Table 4. No significant interactions were observed ( $p$ values for interaction terms $>0.05$ ). In addition, ORs for the association between STF and diabetes development were estimated by re-running model 4 , Table 4 for men and women separately. The results were similar for each sex (OR [95\% CI] men - 1.0 [0.7, 1.5], women $0.8[0.5,1.4]$ ). We also included a BMI $\times$ STF interaction term in models 2 and 3 in Table 4 to determine whether the association between thigh fat and diabetes risk might differ across the spectrum of overall adiposity. In neither model was the interaction term significant (model 2, $p=0.642$, model 3, $p=0.414)$.

\section{Discussion}

In this study we failed to find a significant association between STF area and cumulative incidence of type 2 diabetes. This finding was also seen consistently in unadjusted analyses and multivariable models adjusted for age, sex, family history of diabetes, and overall and regional measures of adiposity. In adjusted models, TC was borderline significantly associated with diabetes incidence in the same direction as seen in other research. Greater TC was associated with a lower odds of incident diabetes in some models, suggesting that fat in this depot may exert a protective effect. Research to resolve the discordance between our findings and existing literature on TC and diabetes risk is needed. As a negative association between STF area and the metabolic syndrome prevalence among obese individuals has been reported in a crosssectional study, we examined whether the association between this fat depot and diabetes risk varied by degree of overall adiposity as measured by BMI in our population [15]. We did not find this to be true, as our results support a negative association between diabetes risk and STF area across the BMI spectrum observed among our participants.

IAF area, on the other hand, was strongly associated with the risk of this outcome. Therefore, this study confirms the important role of visceral adiposity in the development of type 2 diabetes and provides evidence that STF is not independently associated with type 2 diabetes risk. The higher risk associated with greater IAF area was independent of BMI and STF and SAF areas. In this paper we provide additional follow-up to our previous analysis of abdominal adipose areas in which most participants had only completed 5 years of follow-up [10]. The results from the previous analysis of these data showed a similar 
association between abdominal fat depots and type 2 diabetes risk but did not examine the role of thigh fat area.

Cross-sectional studies have reported an association between TC, fat area and type 2 diabetes [1, 2]. However, no report to our knowledge has prospectively examined the relationship between STF measured by CT and type 2 diabetes incidence while adjusting for direct measurements of other significant fat depots. Results from cross-sectional studies have shown inconsistent results in the prediction of diabetes frequency using varied methods of estimating thigh fat $[2,9]$. One study reported greater lower body subcutaneous fat, measured by thigh skinfold thickness, to be associated with a lower likelihood of the presence of type 2 diabetes and insulin resistance, but no adjustment was performed for other regional body fat depots [2]. Using CT, another cross-sectional study reported that individuals with type 2 diabetes and impaired glucose tolerance had similar amounts of STF compared with those with normal glucose tolerance [9]. A major deficiency of such crosssectional research is the inability to ascertain whether higher or lower measures of thigh fat preceded or followed the development of diabetes, insulin resistance or other states of dysglycaemia. Uncertainty of this temporal sequence prohibits definite conclusions to be made from cross-sectional research about whether the size of a fat depot may be causally related to diabetes or other outcomes.

A population-based prospective cohort study examined the relationship between TC and type 2 diabetes risk [6]. Logistic regression models adjusted for BMI, age and waist circumference demonstrated an inverse relationship between type 2 diabetes risk and baseline TC in women only. This study had several limitations including lack of adjustment for regional body fat depots and measurement of TC as a proxy for thigh fat, which is prone to inter-observer variability, and furthermore cannot distinguish between muscle and fat components that contribute to overall circumference [14].

There are limitations to this prospective study. Thirty participants were lost to follow-up due to death, inability to locate or withdrawal from the study; however, we believe our follow-up rate of over $80 \%$ of participants over a period of 10 years makes our observations unlikely to be biased due to these missing data points. Although we adjusted for multiple covariates, the possibility exists that confounding might still be present due to unmeasured covariates. The cohort we studied was restricted to Japanese Americans and therefore it is possible that our observations may not apply to other ethnic groups. Although it may be that our failure to observe an association between thigh fat area and diabetes incidence could be due to insufficient power, we believe that our observations of the previously noted association between TC and diabetes incidence and between IAF area and risk of this outcome suggest that this is unlikely to be the case. CT scans of the thigh in this study focused on subcutaneous fat area only and did not define inter-muscular fat content, which has been shown to be related to metabolic abnormalities in post-menopausal women, although this depot is of much lesser importance than visceral fat [16]. We were also unable to examine thigh muscle area by CT scan as a possible explanation for the inverse association others have noted between TC and diabetes risk, as this measurement was not performed from the original CT images, which are no longer available for additional study. Higher muscle mass has been reported in association with lower glucose and insulin concentrations, and may help to explain the correlation between greater TC and lower diabetes risk [17]. To our knowledge, no prospective investigation of the risk of type 2 diabetes in relation to thigh muscle mass or inter-muscular fat has been conducted. In addition, our population was on average of normal weight with a mean BMI of $24.1 \mathrm{~kg} / \mathrm{m}^{2}$. Whether these findings would hold for heavier populations is not known.

We did not directly measure body fat but instead STF, SAF and IAF measurements were each determined by a single CT slice. A high correlation between a single CT scan slice and direct measurement of visceral fat volume has been shown, which limits the potential for bias [18, 19]. No studies to our knowledge have examined the correlation between a single CT scan slice and direct measurement of thigh fat volume. It has been shown using dual-energy $\mathrm{x}$ ray absorptiometry measures of thigh fat that these correlate well with adipose tissue mass measured by multislice CT techniques [20]. However, the correlation between a single CT scan slice and STF volume remains unexplored. Adjustment for total adiposity was performed using BMI. This represents an indirect measure of adiposity and may not have resulted in complete adjustment for body fat mass.

In conclusion, we found no evidence that directly measured STF was related to diabetes incidence despite the finding of an inverse borderline significant association between TC and diabetes risk in our population. The concept of the 'metabolically healthy' thigh fat depot is not supported by our data. We have again demonstrated the importance of IAF area as the most important fat depot predictor of the development of type 2 diabetes. These observations provide evidence that IAF remains the key fat depot related to type 2 diabetes risk among Japanese Americans, whereas thigh fat area does not appear to have a role in the development of this outcome.

Acknowledgements This work was supported by National Institutes of Health Grants DK-31170, HL-49293 and DK-02654; by facilities and services provided by the Diabetes and Endocrinology Research Center (DK-17047), Clinical Nutrition Research Unit (DK-35816), and the General Clinical Research Center (RR-00037) at the University of Washington. VA Puget Sound Health Care System provided support for the involvement of E.J. Boyko and S.E. Kahn in this research. We are grateful to the King County Japanese-American community for their support and cooperation. 
Contribution statement DH participated in the conception and design, analysed and interpreted the data, drafted the manuscript and provided final approval of the version to be published. EJB, MJM, DLL, SEK and WYF participated in the conception and design, revised the paper for important intellectual content and provided final approval of the version to be published.

Duality of interest The authors declare that there is no duality of interest associated with this manuscript.

\section{References}

1. Chuang YC, Hsu KH, Hwang CJ, Hu PM, Lin TM, Chiou WK (2006) Waist-to-thigh ratio can also be a better indicator associated with type 2 diabetes than traditional anthropometrical measurements in Taiwan population. Ann Epidemiol 16:321-331

2. Livingston EH (2006) Lower body subcutaneous fat accumulation and diabetes mellitus risk. Surg Obes Relat Dis 2:362-368

3. Snijder MB, Visser M, Dekker JM et al (2005) Low subcutaneous thigh fat is a risk factor for unfavourable glucose and lipid levels, independently of high abdominal fat. The Health ABC Study. Diabetologia 48:301-308

4. Aasen G, Fagertun H, Halse J (2008) Regional fat mass by DXA: high leg fat mass attenuates the relative risk of insulin resistance and dyslipidaemia in obese but not in overweight postmenopausal women. Scand J Clin Lab Invest 68:204-211

5. Aasen G, Fagertun H, Halse J (2009) Insulin resistance and dyslipidaemia in obese premenopausal and postmenopausal women matched for leg/trunk fat mass ratio. Scand J Clin Lab Invest 69:505-511

6. Snijder MB, Dekker JM, Visser M et al (2003) Associations of hip and thigh circumferences independent of waist circumference with the incidence of type 2 diabetes: the Hoorn Study. Am J Clin Nutr 77:1192-1197

7. Manolopoulos KN, Karpe F, Frayn KN (2010) Gluteofemoral body fat as a determinant of metabolic health. Int J Obes 34:949959

8. Tulloch-Reid MK, Williams DE, Looker HC, Hanson RL, Knowler WC (2003) Do measures of body fat distribution provide information on the risk of type 2 diabetes in addition to measures of general obesity? Comparison of anthropometric predictors of type 2 diabetes in Pima Indians. Diabetes Care 26:2556-2561

9. Goodpaster BH, Krishnaswami S, Resnick H et al (2003) Association between regional adipose tissue distribution and both type 2 diabetes and impaired glucose tolerance in elderly men and women. Diabetes Care 26:372-379

10. Boyko EJ, Fujimoto WY, Leonetti DL, Newell-Morris L (2000) Visceral adiposity and risk of type 2 diabetes: a prospective study among Japanese Americans. Diabetes Care 23:465-471

11. Fox CS, Massaro JM, Hoffmann U et al (2007) Abdominal visceral and subcutaneous adipose tissue compartments: association with metabolic risk factors in the Framingham Heart Study. Circulation 116:39-48

12. Ross R, Fortier L, Hudson R (1996) Separate associations between visceral and subcutaneous adipose tissue distribution, insulin and glucose levels in obese women. Diabetes Care 19:1404-1411

13. Fujimoto WY, Leonetti DL, Kinyoun JL et al (1987) Prevalence of diabetes mellitus and impaired glucose tolerance among secondgeneration Japanese-American men. Diabetes 36:721-729

14. Shuman WP, Morris LL, Leonetti DL et al (1986) Abnormal body fat distribution detected by computed tomography in diabetic men. Invest Radiol 21:483-487

15. Goodpaster BH, Krishnaswami S, Harris TB et al (2005) Obesity, regional body fat distribution, and the metabolic syndrome in older men and women. Arch Intern Med 165:777-783

16. Dube MC, Lemieux S, Piche ME et al (2011) The contribution of visceral adiposity and mid-thigh fat-rich muscle to the metabolic profile in postmenopausal women. Obesity 19:953-959

17. Kalyani RR, Metter EJ, Ramachandran R, Chia CW, Saudek CD, Ferrucci L (2011) Glucose and insulin measurements from the oral glucose tolerance test and relationship to muscle mass. J Gerontol A Biol Sci Med Sci. doi:10.1093/gerona/glr022

18. Han TS, Kelly IE, Walsh K, Greene RM, Lean ME (1997) Relationship between volumes and areas from single transverse scans of intra-abdominal fat measured by magnetic resonance imaging. Int J Obes Relat Metab Disord 21:1161-1166

19. Schoen RE, Thaete FL, Sankey SS, Weissfeld JL, Kuller LH (1998) Sagittal diameter in comparison with single slice CT as a predictor of total visceral adipose tissue volume. Int J Obes Relat Metab Disord 22:338-342

20. Levine JA, Abboud L, Barry M, Reed JE, Sheedy PF, Jensen MD (2000) Measuring leg muscle and fat mass in humans: comparison of CT and dual-energy X-ray absorptiometry. J Appl Physiol $88: 452-456$ 